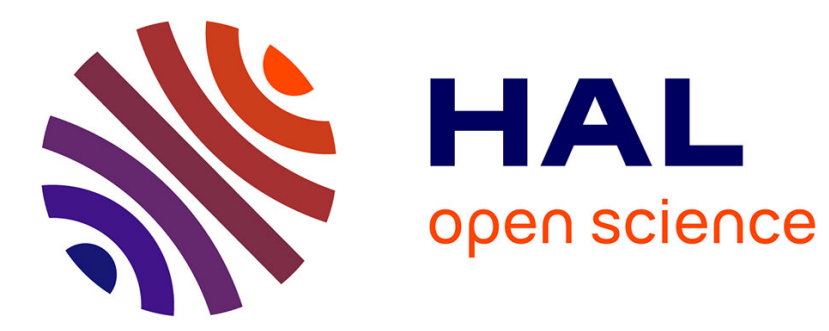

\title{
Étude des solides par annihilation de positons
}

Ch. Janot

\section{To cite this version:}

Ch. Janot. Étude des solides par annihilation de positons. Revue de Physique Appliquée, 1976, 11 (1), pp.89-100. 10.1051/rphysap:0197600110108900 . jpa-00244048

\section{HAL Id: jpa-00244048 https://hal.science/jpa-00244048}

Submitted on 1 Jan 1976

HAL is a multi-disciplinary open access archive for the deposit and dissemination of scientific research documents, whether they are published or not. The documents may come from teaching and research institutions in France or abroad, or from public or private research centers.
L'archive ouverte pluridisciplinaire HAL, est destinée au dépôt et à la diffusion de documents scientifiques de niveau recherche, publiés ou non, émanant des établissements d'enseignement et de recherche français ou étrangers, des laboratoires publics ou privés. 


\title{
ÉTUDE DES SOLIDES PAR ANNIHILATION DE POSITONS
}

\author{
Ch. JANOT \\ Laboratoire de Physique du Solide (L. A. no 155) \\ Université de Nancy I, C. O. no 140, 54037 Nancy-Cedex, France
}

\begin{abstract}
Résumé. - Les paramètres d'annihilation des positons dans la matière s'expriment directement en fonction des caractéristiques des électrons qui participent à l'annihilation. Dans les solides parfaits, l'expérience peut donc donner des informations sur la densité électronique, la quantité de mouvements des électrons et les structures de bande. La présence d'imperfections (lacunes, dislocations, impuretés, surfaces) modifie ces paramètres dont l'évolution constitue donc une traduction des propriétés des défauts de réseau. Cependant, des problèmes théoriques et expérimentaux non totalement résolus de façon satisfaisante limitent parfois les conclusions à un aspect assez qualitatif.
\end{abstract}

Abstract. - The annihilation parameters of positrons in matter can be expressed as functions of the characteristics of electrons which are involved in the annihilation process. Then, in perfect solids, experiments may lead to useful information about electronic density, electron momentum and band structure. In solids containing lattice defects - vacancies, dislocations, impurities, surfaces... - these parameters are sensitively changed so that positron annihilation turns to be a promising method of studying imperfect crystals. However, both theoretical and experimental efforts are still needed to go further on than the sometimes purely qualitative aspect of the results which are obtained.

1. Introduction. - Le positon est l'antiparticule de l'électron dont, par conséquent, il possède la masse $\left(m_{0} c^{2}=0,511 \mathrm{MeV}\right)$; sa charge est égale à $+\mathrm{e}$ et il possède un spin $1 / 2$, avec un moment magnétique parallèle.

Si un positon pénètre dans la matière, il est rapidement thermalisé par collisions élastiques et son énergie décroît jusqu'à une valeur de l'ordre de $k_{\mathrm{B}} T$. Le temps de thermalisation dépend du type de matériau ; il est de l'ordre de $10^{-12} \mathrm{~s}$ dans les solides. Après quoi, le positon s'annihile avec un électron assez éloigné des ions positifs qui repoussent les positons par interactions coulombiennes; ainsi, on peut prévoir que l'annihilation se fera préférentiellement avec les électrons de conduction dans les métaux (de préférence au voisinage de zones lacunaires ou d'impuretés de plus faible valence) avec des électrons extérieurs des ions négatifs dans les cristaux ioniques, et près des régions de faible densité dans les solides moléculaires.

Dans la plupart des cas, l'annihilation d'une paire positon-électron se fait avec l'émission de deux photons $\gamma$. Les paramètres caractéristiques du processus d'annihilation sont :

- la durée de vie du positon,

- la corrélation angulaire des deux photons $\gamma$,

- le spectre d'énergie des photons $\gamma$.

Ces paramètres sont sensibles à la structure électronique (densité et symétries) et fortement influencés par la présence d'imperfection dans le cas de solides cristallisés. Plusieurs articles de synthèses ont été publiés assez récemment $[1,2,3,4,5,6]$; outre une information scientifique détaillée, ils contiennent un nombre impressionnant de références à des articles originaux que nous ne citerons pas ici en détail. Nous nous limiterons à une brève description des bases physiques permettant d'utiliser l'annihilation de positons dans l'étude de l'état solide, en mettant l'accent sur les problèmes qui, semble-t-il, restent imparfaitement résolus pour que la méthode puisse être considérée comme rigoureusement quantitative en toutes circonstances.

2. Paramètres caractéristiques de l'annihilation d'une paire positon-électron. - 2.1 LES DIFFÉRENTS PROCESSUS D'ANNIHILATION. - 2.1.1 Annihilation libre. L'annihilation d'une paire positon-électron se fait avec émission de photons dont l'énergie doit être de l'ordre de $m_{0} c^{2}=0,511 \mathrm{MeV}$, bien supérieure à l'énergie initiale de l'électron (quelques $\mathrm{eV}$ ) et du positon (une fraction de $\mathrm{eV}$ ). Le centre de masse du système peut donc en première analyse être considéré comme au repos et doit le rester après l'annihilation, ce qui implique l'émission d'au moins deux photons si aucune autre particule ne participe à la réaction.

L'accroissement d'une unité dans le nombre de photons émis ou absorbés implique que la section efficace pour le processus est multipliée par un facteur 
de l'ordre de grandeur de la constante de structure fine

$$
\alpha=\frac{e^{2}}{\hbar c}=\frac{1}{137} .
$$

Le calcul exact indique que la section efficace $\sigma_{3 \gamma}$ pour le processus à 3 phonons est inférieure à $0,3 \%$ de la section efficace $\sigma_{2 \gamma}$ pour le processus à deux photons.

On peut aussi envisager une annihilation avec émission d'un seul photon, à condition qu'un troisième corps de masse $M$ (noyau ou électron) participe au processus pour absorber le moment de recul. La section efficace doit alors être multipliée par un facteur de l'ordre de $\lambda_{\mathrm{c}}{ }^{3} \rho$ où $\lambda_{\mathrm{c}}$ est la longueur d'onde Compton de l'électron et $\rho$ la densité du milieu qui absorbe le moment de recul. Il en résulte que $\sigma_{\gamma} / \sigma_{2 \gamma}$ reste inférieure à $10^{-9}$.

En conclusion, l'annihilation libre d'une paire électron-positon se fait pratiquement toujours avec émission de deux photons $\gamma$.

2.1.2 Etat lié du positon: le positonium (Ps). Le positon peut former un état lié avec un électron avant l'annihilation. La structure obtenue est alors analogue à celle d'un atome d'hydrogène et est appelée positonium de symbole Ps.

Ses caractéristiques sont bien décrites par le modèle simple de Bohr, avec une masse réduite égale à $m / 2$, donc une énergie d'ionisation de $6,8 \mathrm{eV}$ et un rayon de $1,06 \AA$ pour la première orbite.

Le Ps peut exister sous deux états :

- un état triplet ${ }^{3} \mathrm{~S}_{1}$ ou ortho-positonium où les spins de l'électron et du positon sont parallèles,

- et un état singulet ${ }^{1} \mathrm{~S}_{0}$ ou para-positonium où les spins sont anti-parallèles.

La conservation de la parité permet de connaître le nombre $n$ de photons émis lorsque le Ps est détruit. On a en effet :

$$
P_{\mathrm{c}}=P_{\mathrm{I}} \cdot P_{l} \cdot P_{\sigma}
$$

où $P_{\mathrm{c}}=(-1)^{n}$ est la parité de charge d'un système contenant $n$ photons, $P_{\mathrm{I}}=-1$ est la parité interne de la paire d'antiparticules, $P_{l}=(-1)^{l}=+1$ est la parité orbitale et $P_{\sigma}=-(-1)^{\sigma}$ est la parité de spin ( -1 pour le para-Ps et +1 pour l'ortho-Ps). Il en résulte que :

$$
(-1)^{n}=(-1) \cdot(+1)(-1)=+1
$$

( $n$ pair pour l'annihilation du p.Ps) et

$$
(-1)^{n}=(-1)(+1)(+1)=-1
$$

( $n$ impair pour l'annihilation du o.Ps).

Pratiquement, le para-positonium $\left({ }^{1} \mathrm{~S}_{0}\right)$ s'annihile avec émission de deux photons $\gamma$ et l'ortho-positonium $\left({ }^{3} \mathrm{~S}_{1}\right)$ avec émission de trois photons $\gamma$.

(Notons que, pour les mêmes raisons de conserva- tion de la parité, l'annihilation libre à $2 \gamma$ des positons se fera avec des électrons de spin opposé.)

Théoriquement, l'o.Ps est trois fois plus abondant que le p.Ps, mais certains processus de conversion peuvent modifier les intensités relatives des annihilations à $2 \gamma$ et à $3 \gamma$ : annihilation prématurée de l'o.Ps avec un électron autre que celui de l'atome (effet pick-off), échange de l'électron du Ps avec l'électron célibataire d'une molécule', paramagnétique (effet de quenching), action d'un champ magnétique qui provoque le mélange des états de nombre quantique magnétique nul par effet Zeeman (la fraction d'annihilation à $3 \gamma$ est alors réduite de $1 / 3$ et les annihilations à $2 \gamma$ sont doublées).

Le positonium ne peut se former que si les conditions énergétiques sont favorables. Le schéma de la figure 1

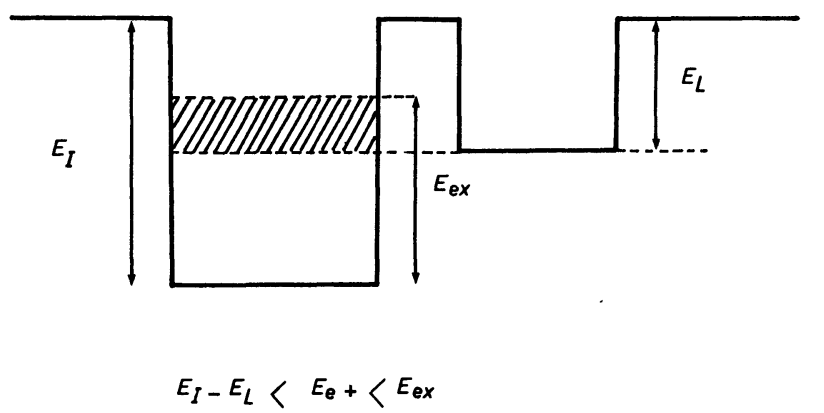

Fig. 1. - Représentation schématique des conditions énergétiques de formation du positonium.

permet de décrire simplement ces conditions. Soit $E_{\mathrm{e}}^{+}$ l'énergie du positon au moment où il rencontre un électron supposé dans un état lié de profondeur $E_{\mathrm{I}}$; $E_{\mathrm{e}}^{+}$doit être suffisante pour faire passer l'électron du puits de profondeur $E_{\mathrm{I}}$ dans un puits de profondeur $E_{\mathrm{L}} \sim 6,8 \mathrm{eV}+\varepsilon$ représentant l'énergie de liaison du positonium augmentée de l'affinité du matériau pour le Ps, donc $E_{\mathrm{e}}^{+}>E_{\mathrm{I}}-E_{\mathrm{L}}$; mais $E_{\mathrm{e}}^{+}$doit rester inférieure à $E_{\mathrm{I}}$ et même inférieure à $E_{\text {ex }}$, l'énergie du premier état excité de l'électron dans son puits, donc finalement :

$$
E_{\mathrm{I}}-E_{\mathrm{L}}<E_{\mathrm{e}}^{+}<E_{\mathrm{ex}} .
$$

Dans les cristaux moléculaires, $E_{\mathrm{ex}}$ et $E_{\mathrm{I}}$ peuvent être très voisins et la formation de Ps est énergétiquement possible, ainsi que dans les gaz. Mais dans les cristaux ioniques et surtout dans les métaux, on aura pratiquement toujours $E_{\mathrm{ex}} \lesssim E_{\mathrm{I}}-E_{\mathrm{L}}$ et la condition de formation du Ps sera impossible à satisfaire, sauf pour les états de surface où $E_{\mathrm{I}}$ peut être très faible.

2.1.3 Autres états liés ou piégés du positon. - Le positon peut aussi s'annihiler dans des états dits piégés, lorsque telle région d'un solide se montre plus particulièrement attractive, parce que la densité locale de charges négatives y est plus forte que la moyenne. Ces annihilations localisées peuvent se 


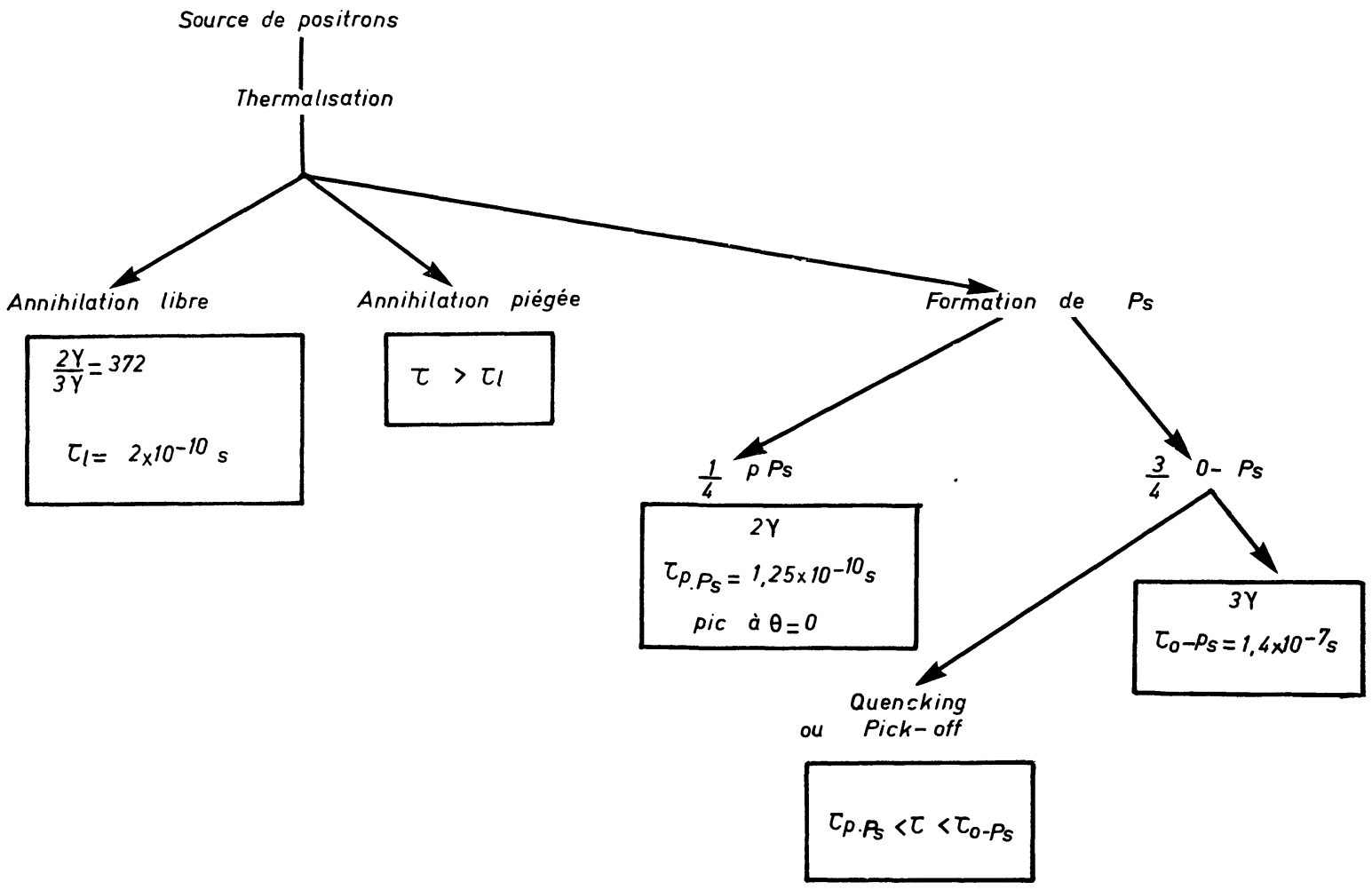

FIg. 2. - Principaux processus d'annihilation du positon.

produire par exemple près des ions négatifs, des lacunes d'ions positifs, des interstitiels d'ions négatifs en ce qui concerne les cristaux ioniques, et près des défauts lacunaires en général dans les solides. Comme pour l'annihilation libre, et pour les mêmes raisons, les états piégés s'annihilent pratiquement avec émission de 2 photons $\gamma$.

La figure 2 résume ces différentes situations, avec leurs caractéristiques essentielles. Nous retiendrons que dans la plupart des cas, l'annihilation dans les solides se fait librement ou en états piégés, suivant un processus à $2 \gamma$. C'est surtout ce processus que nous allons analyser maintenant avec quelques détails.

2.2 TeMPS DE VIE DU POSITON. - Dans un processus à $2 \gamma$, le taux d'annihilation $\lambda_{2 \gamma}$ ou nombre moyen relatif d'annihilation par unité de temps dans l'unité de volume, peut être exprimé en théorie cinétique simple par :

$$
\lambda_{2 \gamma}=\sigma_{2 \gamma} v n_{-}
$$

où $v$ est la vitesse relative des deux particules et $n_{-}$le nombre d'électrons par unité de volume au point d'annihilation. Le calcul exact requiert la connaissance des fonctions d'onde du positon et des électrons en interactions entre eux et avec le milieu. Nous reviendrons plus loin sur cette question dont l'approche dépend en fait du type de matériau où a lieu l'annihilation.

La section efficace $\sigma_{2 \gamma}$ pour un positon libre et un électron dans un état stationnaire a été calculée par Dirac :

$$
\begin{aligned}
\sigma_{2 \gamma} & =\frac{\pi r_{0}^{2}}{\gamma+1} \times \\
& \times\left\{\frac{\gamma^{2}+4 \gamma-1}{\gamma^{2}-1} \log \left[\gamma+\left(\gamma^{2}-1\right)^{1 / 2}\right]-\frac{\gamma+3}{\left(\gamma^{2}-1\right)^{1 / 2}}\right\}
\end{aligned}
$$

avec

$$
\gamma=\left(1-\frac{v^{2}}{c^{2}}\right)^{-1 / 2}
$$

et

$$
r_{0}=\frac{e^{2}}{m_{0} c^{2}}
$$

le rayon classique de l'électron.

Comme $v$ reste très faible devant la vitesse de la lumière, on a une bonne estimation en prenant :

$$
\sigma_{2 \gamma}=\frac{\pi r_{0}^{2} c}{v}
$$

soit

$$
\lambda_{2 \gamma}=\pi r_{0}^{2} c n_{-} \text {. }
$$

La durée de vie du positon est alors définie par

$$
\tau_{2 \gamma}=\frac{1}{\lambda_{2 \gamma}}=\frac{1}{\pi r_{0}^{2} c n_{-}} .
$$


Même dans les milieux de densité électronique très élevée comme les métaux, on obtient des valeurs pour $\tau_{2 \gamma} \sim 10^{-10} \mathrm{~s}$, très supérieures au temps de thermalisation du positon $\left(10^{-12} \mathrm{~s}\right)$. En annihilation libre ou piégée, on peut donc considérer que le positon est au repos et que $v$ représente la vitesse de l'électron.

Pour le positonium, la fonction d'onde dans l'état fondamental s'écrit

$$
\psi_{\mathrm{Ps}}(r)=\left(\pi a^{3}\right)^{-1 / 2} \exp (-r / a)
$$

avec

$$
a=\frac{2 \hbar^{2}}{m_{0} e^{2}}=1,06 \AA
$$

ce qui permet de calculer $n_{-}$pour $r=0$ de façon exacte.

La relation (i) se réduit alors à :

$$
\lambda_{\mathrm{p} . \mathrm{Ps}}=\frac{4 r_{0}^{2} c}{a^{3}}
$$

soit une durée de vie du para-positonium

$$
\tau_{\mathrm{p} . \mathrm{Ps}}=1,25 \times 10^{-10} \mathrm{~s} .
$$

La durée de vie de l'ortho-positonium peut être déduite du rapport des sections efficaces $\sigma_{3 \gamma} / \sigma_{2 \gamma}$; on obtient ainsi :

$$
\sigma_{\mathrm{o} . \mathrm{Ps}}=1,4 \times 10^{-7} \mathrm{~s} .
$$

Ces temps de vie du positonium correspondent à une annihilation de l'atome dans le vide et peuvent être sensiblement abrégés par interaction du posito- nium avec la matière. Notons enfin qu'un positonium isolé peut être considéré comme thermalisé, si bien que l'annihilation se fait pratiquement à vitesse nulle du centre de masse du système.

Mesure des temps de vie :

La détection des $\gamma$ qui résultent de l'annihilation indique avec précision l'instant de la disparition du positon. Le temps zéro, ou instant de la naissance du positon, peut être obtenu en détectant le rayonnement $\beta^{+}$ou, mieux, grâce à un rayonnement $\gamma$ émis en cascade avec le $\beta^{+}$à partir d'un niveau excité dont le temps de vie soit de l'ordre de $10^{-12}-10^{-11} \mathrm{~s}$. C'est ce qui se passe en particulier avec la source de positon ${ }^{22} \mathrm{Na}$, dont la figure 3 donne le schéma de désintégration.

L'enregistrement du nombre des coïncidences retardées en fonction du retard entre les $\gamma$ de nais-

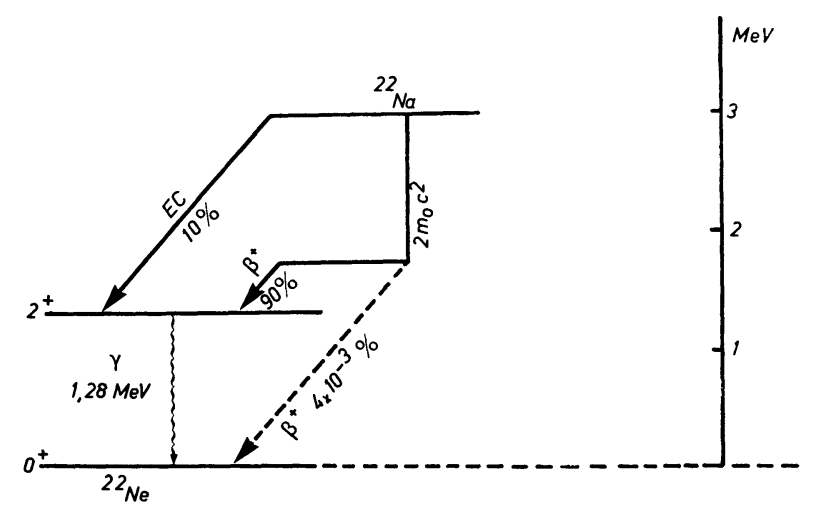

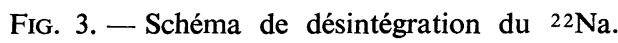

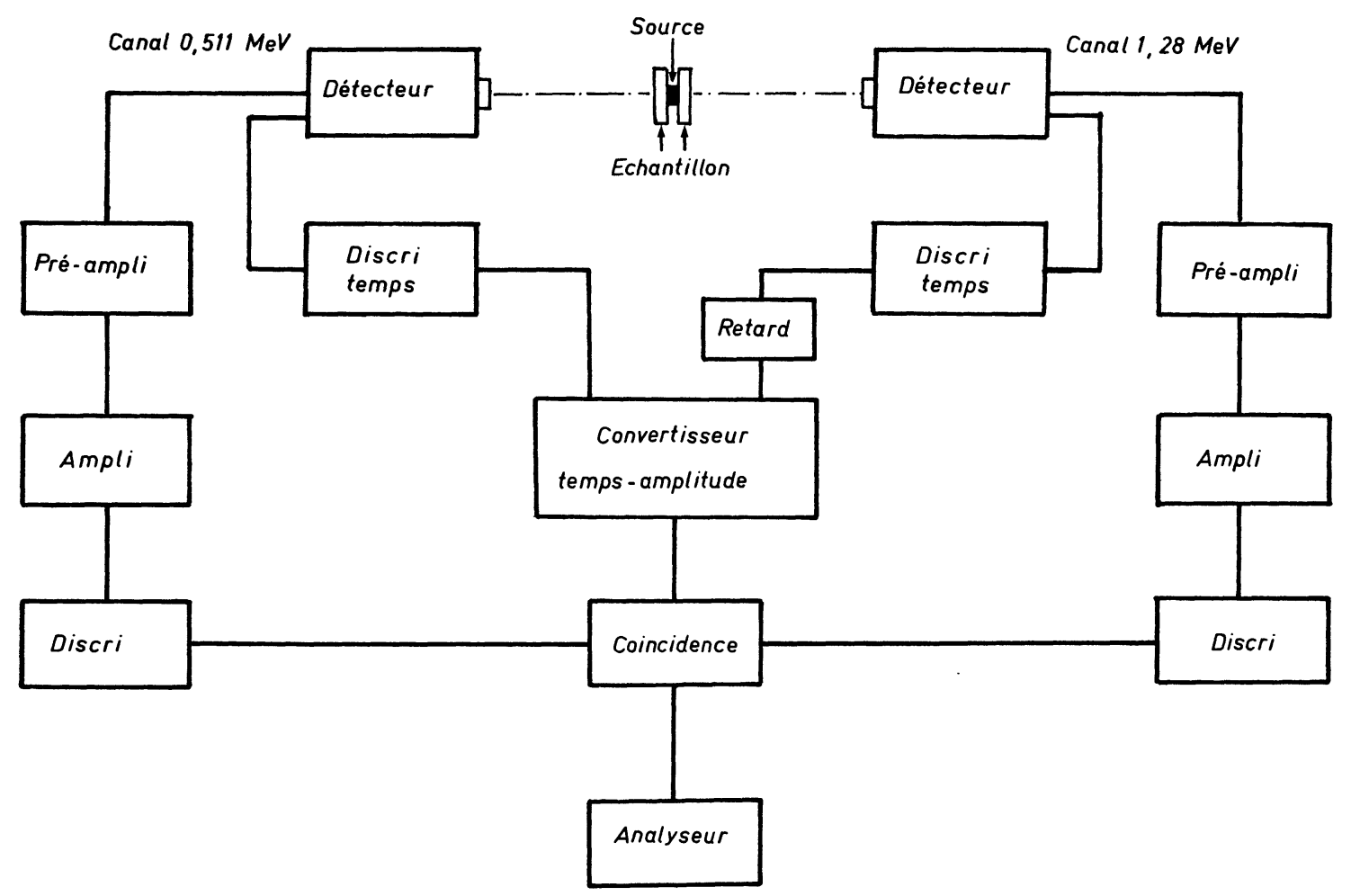

Fig. 4. - Schéma d'un dispositif de mesure des temps de vie du positon. 
sance $(1,28 \mathrm{MeV})$ et les $\gamma$ d'annihilation $(0,511 \mathrm{MeV})$ détectés par deux compteurs fournit le spectre de temps de vie du positon. La figure 4 donne le schéma de principe d'un dispositif de mesure du temps de vie.

S'il existe un seul processus d'annihilation caractérisé par un taux $\lambda$, la loi de répartition des annihilations dans le temps s'écrit :

$$
\frac{\mathrm{d} N}{\mathrm{~d} t}=-\lambda N=-\lambda N_{0} \mathrm{e}^{-\lambda t}
$$

En représentation semi-logarithmique, la courbe $N(t)$ est une droite de pente $\lambda=\tau^{-1}$. Cependant, ce calcul suppose une résolution parfaite du système de détection. En pratique, la courbe obtenue doit être analysée comme le produit de convolution de $N(t)$ par la fonction de résolution dans le temps $P(t)$ de l'appareillage.

En présence de plusieurs processus d'annihilation caractérisés par des taux $\lambda_{i}$ différents, le nombre d'annihilations global sera donné par :

$$
\frac{\mathrm{d} N}{\mathrm{~d} t}=-\sum_{i} \lambda_{i} N_{0} \mathrm{e}^{-\lambda_{i} t}
$$

Outre les valeurs des temps de vie $\tau_{i}=\lambda_{i}^{-1}$, la méthode de la pente permet en principe de déterminer les intensités relatives $I_{i}$ donc les proportions relatives des divers processus d'annihilation ; le nombre d'événements enregistrés pour chaque processus est en effet directement proportionnel à la surface $S_{i}$ située sous la droite qui lui correspond dans le spectre de temps de vie. La figure 5 montre le type de courbes $N(t)$ et $P(t)$ obtenues généralement.

La mesure de temps de vie de l'ordre de $10^{-10} \mathrm{~s}$ est actuellement presque à la limite des performances techniques. L'amélioration dans ce domaine condi-
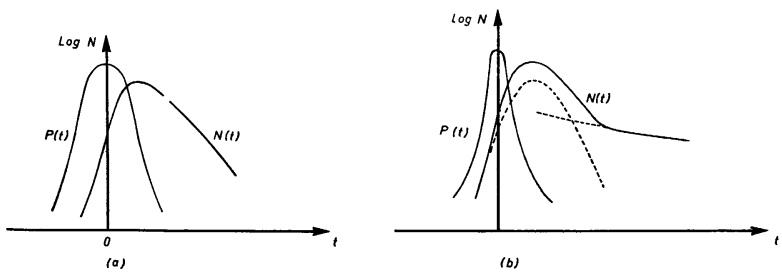

Fig. 5. - Formes typiques de spectres de temps de vie du positon $N(t) ; P(t)$ est la courbe de résolution : $a$ ) une seule vie moyenne, $b$ ) deux vies moyennes.

tionne en fait la possibilité d'analyser avec précision les spectres de temps de vie en leurs diverses composantes éventuelles.

\subsection{DistribUtion ANGULAIRE ET ÉNERGÉTIQUE DES} PHOTONS D'ANNIHILATION. - Dans le processus à $2 \gamma$, outre la vie moyenne du positon thermalisé, l'annihilation libre peut être caractérisée par les distributions angulaire et énergétique des photons émis au cours de l'annihilation.

Si l'électron et le positon s'annihilaient au repos, les deux photons $\gamma$ seraient émis strictement à $180^{\circ}$ l'un de l'autre et emporteraient chacun une énergie égale à $m_{0} c^{2}=0,511 \mathrm{MeV}$.

Le positon thermalisé peut bien être considéré comme s'annihilant au repos, mais l'électron possède une quantité de mouvement $p_{-}$, si bien que les deux photons seront émis à $\pi-\theta$ l'un de l'autre et posséderont une énergie $m_{0} c^{2}+\Delta E$ de façon à ce que soient respectées les lois de conservation.

La technique de corrélation angulaire permet de mesurer un taux d'annihilation $N(\theta)$ en fonction de l'angle de divergence $\theta$ entre les deux gammas.

La figure 6 schématise un dispositif de corrélation angulaire. Deux détecteurs, l'un fixe, l'autre mobile

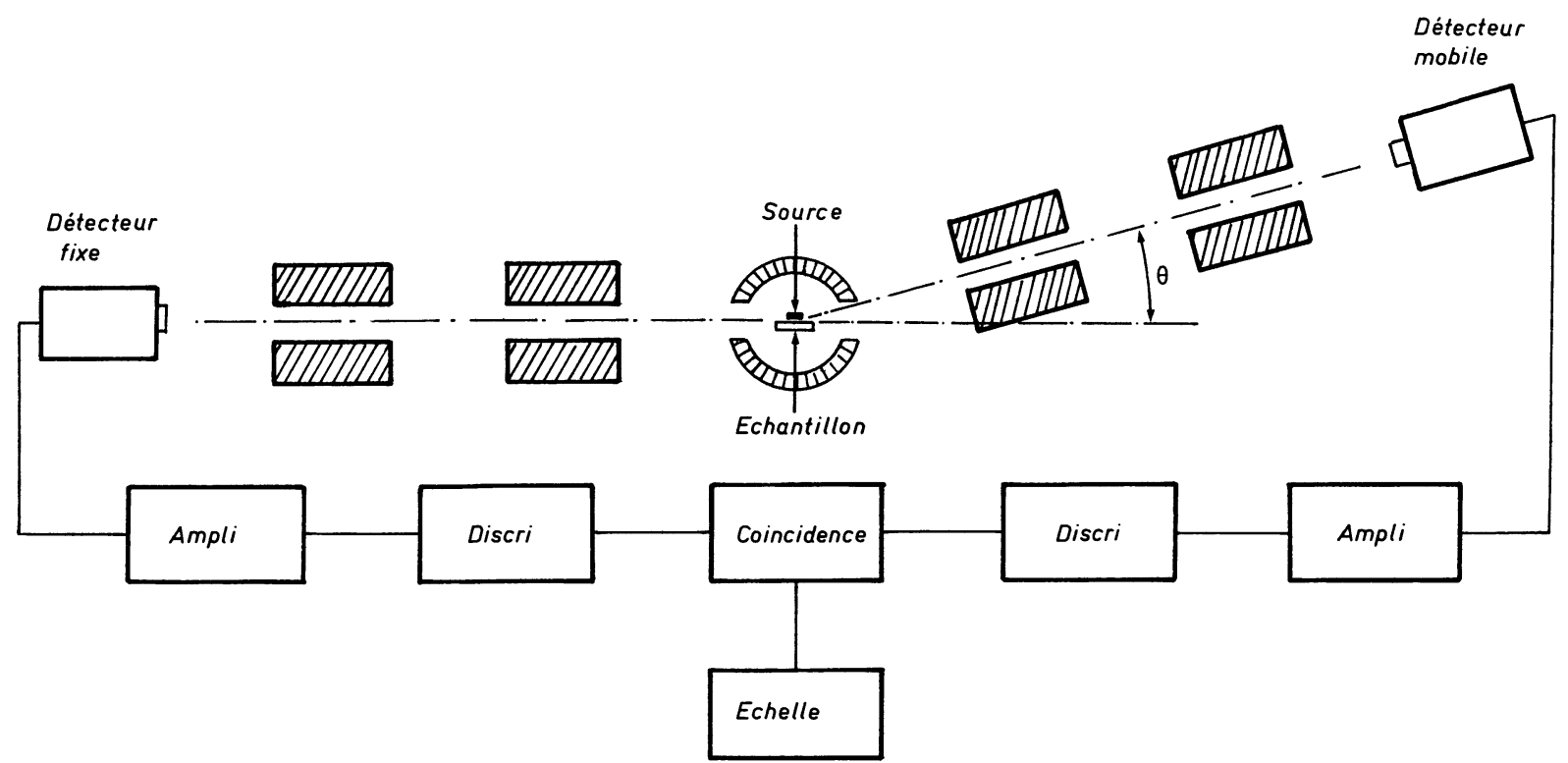

FIg. 6. - Représentation schématique d'un dispositif de corrélation angulaire. 
en rotation, sont disposés de part et d'autre de l'ensemble source-échantillon. Deux longues fentes parallèles collimatrices définissent l'angle solide sous lequel est vu l'échantillon par les détecteurs et la résolution $(\sim 0,1$ milli-radian $)$ de l'appareil. On enregistre les coïncidences en fonction de l'angle $\theta$. Comme $p_{-} \sim 10^{-2} m_{0} c, \theta$ varie sur un intervalle de quelques dizaines de milli-radians.

La figure 7 indique comment $\theta$ peut être relié à la composante $P_{z}$ de la quantité de mouvement de

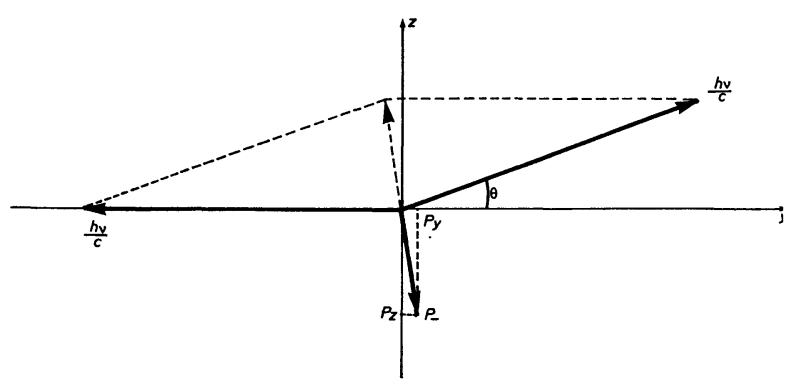

FIG. 7. - Expression de la conservation de la quantité de mouvement.

l'électron annihilé, portée par la direction perpendiculaire aux fentes. On a

$$
P_{z} \simeq m_{0} c \theta \text {. }
$$

On peut donc dire que le nombre de coïncidences $N(\theta)$ correspond au nombre d'électrons annihilés avec une composante $P_{z}=m_{0} c \theta$, quelles que soient les composantes $P_{x}$ et $P_{y}$, soit

$$
N\left(P_{z}\right)=N(m c \theta)=\int_{-\infty}^{+\infty} \rho(\mathbf{p}) \mathrm{d} p_{x} \mathrm{~d} p_{y}
$$

où $\rho(\mathbf{p})$ est la densité dans l'espace des moments des paires d'annihilation électron-positon.

Si la distribution $\rho(\mathbf{p})$ est isotrope, on a :

$$
N\left(P_{z}\right)=2 \pi \int_{-\infty}^{+\infty} \rho(p) p \mathrm{~d} p .
$$

Dans le cas où, de plus, $\rho(p)=\rho_{0}$ est constante à l'intérieur de la surface de Fermi et nulle à l'extérieur

$$
\begin{aligned}
& N\left(P_{z}\right)=2 \pi \rho_{0} \int_{\mathbf{P}_{z}}^{P_{F}} p d p \\
& N\left(P_{z}\right)=\pi \rho_{0}\left(P_{F}^{2}-P_{z}^{2}\right) .
\end{aligned}
$$

La courbe de corrélation angulaire est alorc une parabole inversée, s'annulant pour l'angle

$$
\theta_{\mathrm{F}}=\frac{\hbar k_{\mathrm{F}}}{m_{0} c}
$$

où $k_{\mathrm{F}}$ est le rayon de la sphère de Fermi. La figure 8 montre l'écart à cette distribution dans le cas d'un échantillon d'aluminium. Dans le cas général, il faut connaître la fonction $\rho(\mathbf{p})$ pour pouvoir interpréter les données de corrélation angulaire.

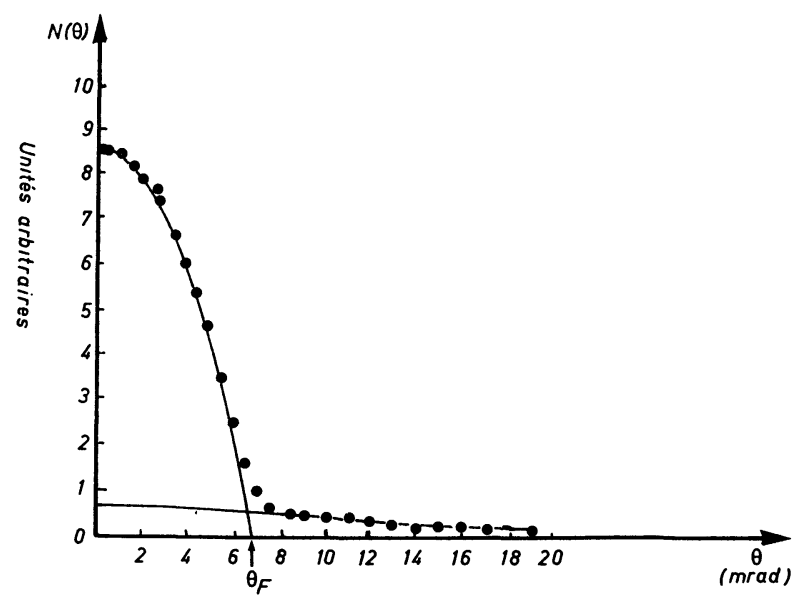

FIG. 8. - Distribution angulaire des photons d'annihilation dans l'aluminium : $\bullet$ points expérimentaux ; - ajustement par une parabole plus une gaussienne.

Remarque : Si l'annihilation à $2 \gamma$ est précédée par la formation d'un atome de p.Ps, la quantité de mouvement de la paire électron-positon est nulle au moment de l'annihilation. La courbe de corrélation angulaire s'enrichit alors d'un pic à $\theta=0$ dont la surface relative donne la proportion de p.Ps formé (Fig. 9).

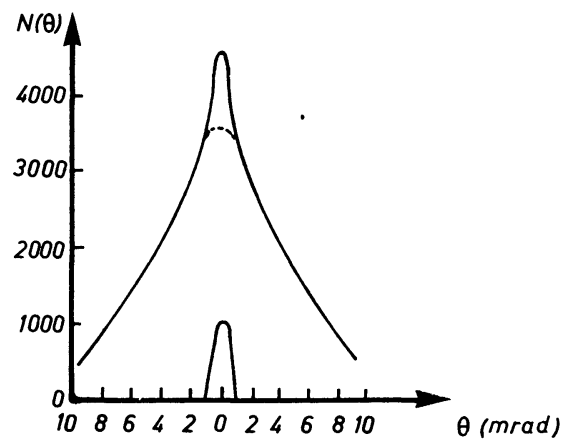

Fig. 9. - Courbe de corrélation angulaire avec composante étroite à $\theta=0$ due à la présence de positonium.

La mesure de la distribution en énergie donne en principe les mêmes informations que les résultats obtenus par corrélation angulaire. Si l'on reprend, en effet, les schémas des figures 6 et 7, la conservation de la quantité de mouvement dans la direction $O y$ de l'émission des photons permet d'écrire :

$$
\frac{h v}{c}=m_{0} c+\frac{P_{y}}{2}
$$

où $P_{y}$ est la composante, dans la direction d'émission des gammas, de la quantité de mouvement de la paire positon-électron avant annihilation. L'énergie des photons est donc

$$
E=m_{0} c^{2}+\frac{c P_{y}}{2}
$$


En mesurant la distribution $N(E)$ des photons émis en fonction de leur énergie, on obtient alors le nombre d'électrons annihilés avec une composante $P_{y}$ déterminée par (iv), quelles que soient les composantes $P_{x}$ et $P_{z}$, soit

$$
N\left(P_{y}\right)=N\left(E-m_{0} c^{2}\right)=\int_{-\infty}^{+\infty} \rho(\mathbf{p}) \mathrm{d} P_{x} \mathrm{~d} P_{z} .
$$

Pour des électrons ayant une énergie de quelque $10 \mathrm{eV}, E-m_{0} c^{2}$ est de l'ordre de $1,5 \mathrm{keV}$. L'utilisation de détecteurs solides $\mathrm{Ge}(\mathrm{Li})$ permet d'avoir une résolution limite voisine de $1 \mathrm{keV}$ pour les énergies de $0,511 \mathrm{MeV}$. Ceci est équivalent à une mesure de corrélation angulaire avec une résolution de quelques milliradians, soit une précision qui est moins bonne d'un ordre de grandeur. En dépit de cela, la technique pourra être préférée dans certaines études à cause de sa grande simplicité de mise en œuvre (un seul détecteur, pas de dispositif mécanique complexe comme en corrélation angulaire), la faible activité des sources nécessaires (quelques $\mathrm{mCi}$ contre plusieurs curies en corrélation angulaire) et la possibilité d'avoir une accumulation rapide des données.

Les progrès passent ici par la mise au point de détecteurs de très grande résolution.

3. Annihilation de positons dans les solides. 3.1 GéNÉRALITÉs. - Dans un solide, le positon peut s'annihiler soit avec des électrons de conduction, soit avec des électrons liés, la première possibilité étant surtout particulière aux systèmes métalliques. En effet, dans les métaux, le positon est fortement repoussé par les ions positifs du réseau, si bien que la probabilité de trouver le positon est maximale dans les espaces compris entre les cœurs des ions où les électrons de conduction se comportent pratiquement comme des électrons libres. Dans les métaux alcalins, l'aluminium ou le gallium, par exemple, les courbes de corrélation angulaire mesurées sont bien des paraboles inversées, en bon accord avec la relation (iii) et les spectres de temps de vie donnent une seule composante avec $\tau \sim 200$ ps. Dans d'autres métaux cependant, tels le cuivre (Fig. 10) ou les métaux de transition, la courbe de corrélation angulaire est en forme de cloche, avec une distribution montrant une queue prolongée dans la région des moments où $p>p_{\mathrm{F}}$. On attribue généralement cette composante large à l'annihilation des positons avec les électrons dit de cour ; cette notion est assez vague et contient en fait toute contribution électronique ne participant pas à la composante parabolique. L'analyse théorique en ce domaine n'est pas satisfaisante et, de ce fait, l'exploitation des courbes de corrélation angulaire est parfois difficile.

Da ns les solides non métalliques, où presque tous les électrons peuvent être considérés comme liés, il n'existe pas de théorie générale permettant d'interpréter la distribution angulaire des gammas d'annihilation.

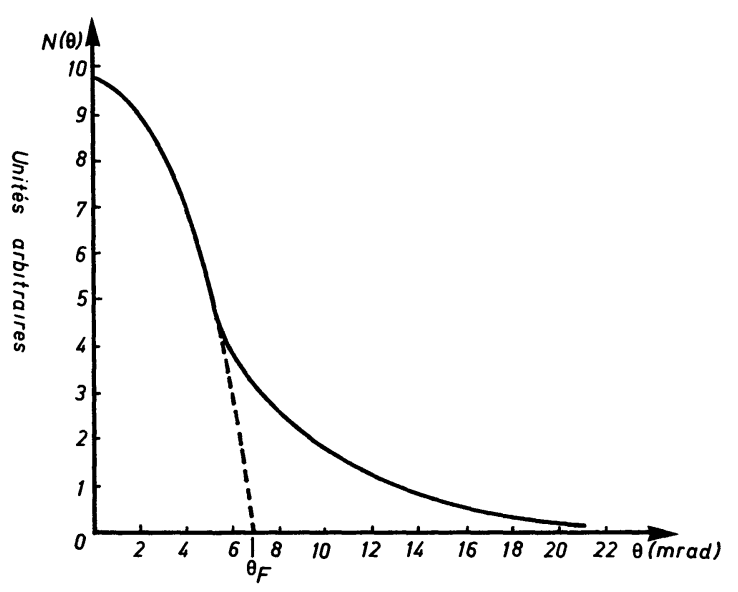

FIG. 10. - Distribution angulaire des photons d'annihilation dans le cuivre.

Les spectres de temps de vie dans les solides dits moléculaires comportent en général trois composantes bien distinctes, allant de 0,1 à 10 ns et qui sont généralement interprétées commes dues à l'annihilation accélérée par effet pick-off d'ortho-positonium pour le temps de vie le plus long, l'annihilation normale de para-positonium pour le temps de vie le plus court, l'annihilation libre pour le temps de vie intermédiaire ; le positonium se formerait dans les espaces intermoléculaires où la densité électronique est très faible. Bien qu'aucune théorie quantitative de ces effets n'existe, la méthode peut être utilisée par exemple dans le cas de changement de phases de polymères à l'état solide, par une sorte de mesure de l'évolution du volume libre intermoléculaire.

Dans les solides ioniques, il existe aussi plusieurs composantes dans le spectre de durée de vie. Mais ici, la formation de positonium est exclue. Les différents temps de vie sont souvent si proches l'un de l'autre que l'analyse des résultats est très difficile. L'ordre de grandeur de ces temps de vie est de 200 ps. Les courbes de corrélation angulaire semblent indiquer que dans des cristaux ioniques sans défauts, le positon s'annihile avec les électrons périphériques des ions négatifs, mais l'hypothèse d'une fonction d'onde du positon sous forme d'onde plane ne peut être totalement rejetée. La localisation ou délocalisation du positon n'est donc pas, ici, un problème résolu.

Le comportement du positon dans un métal sans imperfection fait, comparativement, l'objet d'approches plus précises dont le paragraphe suivant tentera de donner l'essentiel.

\subsection{ANNIHILATION DE POSITONS Dans LeS MÉTAUX. -} Dans la théorie simple qui conduit à l'expression (iii) d'une distribution parabolique pour la courbe de corrélation angulaire, les hypothèses suivantes sont faites :

- l'annihilation se fait uniquement avec les électrons de conduction, 
- ces électrons de conduction sont totalement libres et représentés par une fonction d'onde à une particule du type

$$
\psi_{k}(\mathbf{r})=V^{-1 / 2} \exp \left(i \mathbf{k}_{-} . \mathbf{r}\right),
$$

- le positon est aussi une particule libre, sans interaction ni avec le réseau, ni avec les électrons, dans l'état $k_{+}=0$ et, par conséquent, de fonction d'onde $\psi_{+}(\mathbf{r})=V^{-1 / 2}$.

On peut s'étonner que, même pour les métaux tels que les alcalins ou l'aluminium, l'accord entre un modèle aussi simpliste et l'expérience puisse être somme toute assez satisfaisant. Cet accord n'est qu'apparent :

En effet, de la relation (iii)

$$
N\left(P_{z}\right)=\pi \rho_{0}\left(P_{\mathrm{F}}^{2}-P_{z}^{2}\right)
$$

on peut tirer la probabilité totale d'annihilation

$$
\lambda_{0} \simeq \int_{0}^{P_{F}} N\left(P_{z}\right) \mathrm{d} P_{z}
$$

soit

$$
\lambda_{0}=\tau^{-1} \simeq \frac{2 \pi \rho_{0} P_{\mathrm{F}}^{3}}{3}=12\left(a_{\mathrm{B}} / r_{\mathrm{s}}\right)^{3} 10^{9} \mathrm{~s}^{-1}
$$

où $a_{\mathrm{B}}$ est le rayon de Bohr et $r_{\mathrm{s}}$ le rayon de la sphère correspondant au volume libre occupé par un électron. Ce résultat appliqué à l'aluminium donne $\tau=7,5 \times 10^{-10} \mathrm{~s}$, alors que l'expérience conduit à $1,7 \times 10^{-10} \mathrm{~s}$. Pour les alcalins, le désaccord est encore plus important, comme l'indique la courbe (a) de la figure 11.

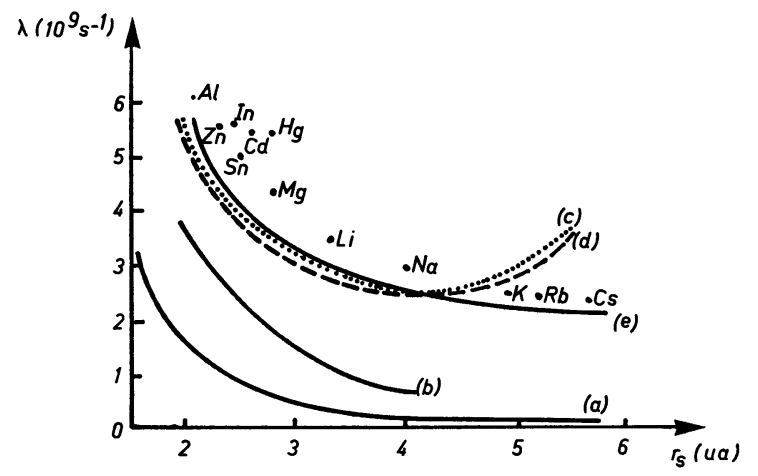

FIG. 11. - Comparaison entre les valeurs mesurées du taux d'annihilation dans les métaux et les estimations théoriques : - points expérimentaux ; a) calcul sans interactions, b) calcul avec interaction directe et écrantage en R. P. A., c) influence des diffusions multiples, $d$ ), e) introduction de la self-consistance.

Un calcul plus précis de $\lambda=\tau^{-1}$ en introduisant successivement différents types d'interactions permet de mesurer l'importance de ces interactions pour la description correcte du positon dans le métal.

En utilisant la notation de la seconde quantifica- tion, la probabilité d'annihilation par unité de temps avec émission d'une paire de photons de moment compris entre $\mathbf{k}$ et $\mathbf{k}+\mathrm{d}^{\mathbf{3}} \mathbf{k}$ est donnée par :

$$
\begin{aligned}
\gamma(\mathbf{k}) \mathrm{d}^{3} \mathbf{k}=\frac{\pi r_{0}^{2} c}{V} \mid \sum_{f} & <f \mid \int \mathrm{d}^{3} \mathbf{x} \exp (-i \mathbf{k} \cdot \mathbf{x}) \times \\
& \times \psi_{-}(\mathbf{x}) \psi_{+}(\mathbf{x})|i>|^{2} \mathrm{~d}^{3} \mathbf{k}
\end{aligned}
$$

où $\psi_{-}(\mathbf{x})$ et $\psi_{+}(\mathbf{x})$ sont les opérateurs de champ d'Heisenberg, $\mid i>$ représente l'état initial de 1 positon et $n$ électron, $\mid f>$ tout état final possible contenant les $n-1$ électrons restant après l'annihilation, $V$ est le volume de normalisation.

En effectuant la somme sur les $|f\rangle$, on obtient

$$
\begin{aligned}
\gamma(\mathbf{k}) \mathrm{d}^{3} \mathbf{k} & =\frac{\pi r_{0}^{2} c}{V} \iint \mathrm{d}^{3} x \mathrm{~d}^{3} y \exp [-i \mathbf{k}(\mathbf{x}-\mathbf{y})] \times \\
& \times<\psi_{-}^{\dagger}(\mathbf{y}) \psi_{+}^{\dagger}(\mathbf{y}) \psi_{+}(\mathbf{x}) \psi_{-}(\mathbf{x})>\mathrm{d}^{3} \mathbf{k} .
\end{aligned}
$$

Le taux d'annihilation totale est obtenu en sommant sur toutes les valeurs de $k$, soit :

$$
\lambda=\frac{\pi r_{0}^{2} c}{V} \int \mathrm{d}^{3} \mathbf{x}<\psi_{-}^{\dagger}(\mathbf{x}) \psi_{-}(\mathbf{x}) \psi_{+}^{\dagger}(\mathbf{x}) \psi_{+}(\mathbf{x})
$$

ce qui exprime bien que la probabilité d'annihilation est proportionnelle à la densité électronique sur le positon, moyennée pour toutes les positions du positon.

Dans le cas simple où l'état de chaque électron est décrit par une fonction $\psi_{i}(\mathbf{r})$ et l'état du positon par une fonction $\psi_{+}(\mathbf{r})$, la probabilité d'annihilation avec le 1 er électron est simplement proportionnelle au carré du module de la transformée de Fourier du produit des fonctions d'onde, soit

$$
\gamma_{i}(\mathbf{k}) \mathrm{d}^{3} \mathbf{k}=\frac{\pi r_{0}^{2} c}{(2 \pi)^{3}}\left|\int \psi_{i}(\mathbf{r}) \cdot \psi_{+}(\mathbf{r}) \mathrm{e}^{-i \mathbf{k} . \mathbf{r}} \mathrm{d}^{3} \mathbf{r}\right|^{2} \mathrm{~d}^{3} \mathbf{k}
$$

La probabilité totale d'annihilation est obtenue en sommant sur tous les états et sur tous les électrons

$$
\begin{aligned}
\lambda & =\sum_{i} \int \gamma_{i}(\mathbf{k}) \mathrm{d}^{3} \mathbf{k} \\
& =\pi r_{0}^{2} c \sum_{i} \int\left|\psi_{i}(\mathbf{r})\right|^{2} \cdot\left|\psi_{+}(\mathbf{r})\right|^{2} \mathrm{~d}^{3} \mathbf{r} .
\end{aligned}
$$

On retrouve alors la valeur de $\lambda_{0}$ si on se place dans le modèle de Sommerfeld avec

$$
\left\{\begin{array}{l}
\psi_{i}(\mathbf{r})=V^{-1 / 2} \exp \left(i \mathbf{k}_{-} \mathbf{r}\right) \\
\psi_{+}(\mathbf{r})=V^{-1 / 2}
\end{array}\right.
$$

On peut reprendre l'expression (viii) de $\lambda$ sous la forme équivalente :

$$
\lambda=i^{2} \pi r_{0}^{2} c \int \mathrm{d}^{3} \mathbf{r} \lim _{t^{\prime} \rightarrow t_{+}} G_{\text {ep }}\left(\mathbf{r} t, \mathbf{r} t ; \mathbf{r} t^{\prime}, \mathbf{r} t^{\prime}\right)
$$


où $G_{\text {ep }}$ est la fonction de Green au zéro absolu de la paire de particules électron-positon

$$
\begin{aligned}
G_{\mathrm{ep}}\left(x, y ; x^{\prime}, y^{\prime}\right) & = \\
= & -i^{2}<T\left\{\psi_{-}(x) \psi_{+}(y) \psi_{+}^{\dagger}\left(y^{\prime}\right) \psi_{-}^{\dagger}\left(x^{\prime}\right)\right\}>
\end{aligned}
$$

que l'on peut écrire

$$
\begin{aligned}
& G_{\mathrm{ep}}\left(x, y ; x^{\prime}, y^{\prime}\right)=G_{\mathrm{e}}^{0}\left(x, x^{\prime}\right) \cdot G_{\mathrm{p}}^{0}\left(y, y^{\prime}\right)+ \\
& \quad+(-i) \int \mathrm{d}^{4} \zeta \cdot \mathrm{d}^{4} \eta \mathrm{d}^{4} \zeta^{\prime} \mathrm{d}^{4} \eta^{\prime} \\
& \quad \times G_{\mathrm{e}}^{0}(x, \zeta) G_{\mathrm{p}}^{0}(y, \eta) I\left(\zeta \eta ; \zeta^{\prime} \eta^{\prime}\right) G_{\mathrm{ep}}\left(\zeta^{\prime} \eta^{\prime} ; x^{\prime}, y^{\prime}\right)
\end{aligned}
$$

où

$$
\begin{aligned}
& G_{\mathrm{e}}^{0}\left(x, x^{\prime}\right)=(-i)<T\left\{\psi_{-}(x) \psi_{-}^{\dagger}\left(x^{\prime}\right)\right\}>0 \\
& G_{\mathrm{p}}^{0}\left(y, y^{\prime}\right)=(-i)<T\left\{\psi_{+}(y) \psi_{+}^{\dagger}\left(y^{\prime}\right)\right\}>0
\end{aligned}
$$

sont les fonctions de Green des particules libres pour le système sans interaction. En ne conservant que le terme $G_{\mathrm{e}}^{0} G_{\mathrm{p}}^{0}$ dans $G_{\mathrm{ep}}$, on obtient le résultat donné par le modèle de Sommerfeld. $I\left(\zeta \eta ; \zeta^{\prime} \eta^{\prime}\right)$ caractérise l'interaction et s'exprime en fonction de $G_{\text {ep }}$ et d'autres propagateurs à plusieurs particules. Sans entrer dans les détails, nous allons signaler successivement les approches qui ont été proposées :

- la première correction consiste à tenir compte de l'interaction directe de Coulomb entre positon et électron, tout en tenant compte de l'écrantage que produit le gaz d'électron environnant. L'effet d'écrantage peut être évalué en sommant les excitations d'un nombre quelconque de paire électron-trou dans le gaz d'électron, par la méthode R. P. A. Cette première correction, qui augmente la densité électronique au voisinage du positon, doit donner un taux d'annihilation $\lambda_{1}>\lambda_{0}$. C'est ce que montre la courbe (b) de la figure 11, calculée numériquement en ne gardant que la correction du premier ordre dans $G_{\text {ep }}$ (c'est-àdire en remplaçant $G_{\mathrm{ep}}$ par $G_{\mathrm{e}}^{0} G_{\mathrm{p}}^{0}$ dans le terme d'interaction) et en tenant compte à la fois de l'écrantage à courte distance et des excitations de plasmon. Il est clair que les valeurs obtenues sont encore beaucoup plus faibles que ce que fournit l'expérience [7] ;

- une seconde correction par rapport à la précédente consiste à tenir compte des diffusions multiples qui peuvent apporter une contribution importante à l'annihilation. Le calcul est difficile à faire, sauf si on se limite au terme statique $u(q, 0)$ de la transformation de Fourier du terme d'interaction dans $G_{\text {ep }}$. On obtient ainsi [8] un taux d'annihilation qui peut se mettre sous la forme :

$$
\lambda_{2}=\lambda_{0} \cdot \varepsilon(p)
$$

avec

$$
\varepsilon(p)=a+b\left(\frac{p}{P_{\mathrm{F}}}\right)^{2}+c\left(\frac{p}{P_{\mathrm{F}}}\right)^{4}
$$

où $a, b$ et $c$ sont coefficients numériques. C'est ce que représente la courbe (c) de la figure 11 ; l'amélioration est sensible, au moins tant que $r_{\mathrm{s}}$ reste inférieur à 5 UA. Pour les plus faibles densités électroniques, le modèle exagère l'effet des diffusions multiples; ceci est certainement dû au fait qu'aucune relation de self-consistance n'est introduite entre les charges déplacées autour du positon et l'écrantage de l'interaction électron-positon.

- Un calcul self-consistant peut être fait en exprimant la constante diélectrique du milieu en fonction du facteur de structure ou transformée de Fourier de la fonction statique de distribution de paire. Le calcul est classique pour un système d'électron. Son extension à un système à deux composantes (positonélectron) [9] donne un résultat très proche du cas précédent (courbe « $d$ » de la Fig. 11). Une amélioration est apportée [10] en tenant compte du changement de la fonction de corrélation de paire sous l'action d'un faible potentiel extérieur. Le résultat est représenté par la courbe «c » de la figure 11.

Il n'existe pas actuellement de théories qui permettent de rendre compte de l'annihilation avec les électrons non libres du métal. On remarque généralement, par une comparaison entre le temps de vie directement mesuré et celui que l'on peut déduire de l'aire située sous les différentes composantes de la courbe de corrélation angulaire, que la différence entre la courbe « e » et les points expérimentaux de la figure 11 correspond bien à la fraction d'annihilation avec les électrons de la composante non parabolique.

Dans le cas d'alliage, le problème est encore compliqué par le fait que la fonction d'onde du positon n'est plus simplement périodique sur le réseau cristallin si l'annihilation se produit préférentiellement sur un type d'atome [11]. Nous rencontrerons un effet similaire dans le cas de cristaux comportant des imperfections.

3. 3 ETUdE DES ÉTATS ÉLECTRONIQUES DANS LES SOLIDES PAR ANNIHILATION DE POSITON. - En dépit de la situation théorique qui vient d'être décrite, des informations de type quantitatif sur les états électroniques dans les solides peuvent être déduites des expériences d'annihilation de positon.

La première information, globale, est la détermination des densités électroniques aux points d'annihilation, à partir de la relation (i), par mesure des temps de vie. Si on sait par ailleurs où se produit l'annihilation, ces densités peuvent alors être attribuées à des points bien précis du solide. Ceci peut être particulièrement intéressant par exemple pour étudier la charge électronique dans des sites interstitiels, ou sur un des composés d'un alliage, etc.

Les courbes de corrélation angulaire donnent le même renseignement à partir de l'aire totale. L'analyse en différentes composantes permet de donner les proportions relatives de différentes classes d'électrons 
(de conduction, de cœur...) et même d'explorer, section par section, l'espace des moments puisque l'amplitude en chaque point de la courbe de corrélation angulaire est fonction du nombre d'états occupés dans un plan qui coupe l'espace réciproque à la distance $p_{z}$ de l'origine, perpendiculairement à la direction d'observation.

3.3.1 Solides moléculaires. - La composante étroite centrée sur $\theta=0$ donne la fraction électronique intermoléculaire caractérisant les liaisons de type Van Der Waals tandis que la composante large est liée à la charge électronique qui participe aux liaisons covalentes.

3.3.2 Solides ioniques. - L'existence d'une faible composante parabolique, comme dans le cas de LiH [12], montre la présence d'électrons délocalisés et mesure son importance. Les résultats ne dépendent que de la nature de l'anion.

3.3.3 Solides métalliques ou semi-conducteurs. La corrélation angulaire permet de tester le modèle de l'électron libre par l'importance relative de la composante parabolique et d'effectuer une mesure du niveau de Fermi ; cette composante est pratiquement unique pour les alcalins, alors qu'une composante large existe pour les métaux nobles, les métaux de transition ou les semi-conducteurs, traduisant une forte contribution de cæur surtout due aux électrons $\mathrm{d}$. A l'intérieur d'une même catégorie, l'effet de valence et de volume de l'ion joue également un rôle très sensible.

La largeur de la composante parabolique mesure la densité d'électron libre. Il y a là un moyen de déterminer la valence du métal, d'étudier des effets de pression ou de remplissage de la bande de conduction par apport d'un élément extérieur $(\mathrm{H}$ dans $\mathrm{Pd}$ ou $\mathrm{Ti}$ par exemple, ou alliages Se-Te).

3.3.4 Matériaux magnétiques. - Les courbes de corrélation angulaire peuvent, dans le cas de matériaux magnétiques, donner une sorte de mesure de la polarisation de spin en même temps qu'une confirmation que la contribution de caur vient surtout des électrons $d$.

Les positons émis durant la désintégration $\beta^{+}$de la source sont partiellement polarisés dans le sens de leur mouvement ; cette polarisation est conservée jusqu'à l'annihilation.

$\mathrm{Si}$ on applique un champ magnétique extérieur à un matériau ferromagnétique, il y a orientation préférentielle des spins des électrons non appariés (par exemple les électrons $3 \mathrm{~d}$ dans les métaux de la première série de transitions). Soit $N(\theta) \uparrow \uparrow$ et $N(\theta) \uparrow \downarrow$ les courbes de corrélations angulaires obtenues suivant que les spins électroniques sont orientés par le champ appliqué parallèlement ou antiparallèlement au faisceau de positons. On doit avoir :

$$
\begin{aligned}
& N(\theta) \downarrow \uparrow>N(\theta) \\
& N(\theta) \uparrow \uparrow<N(\theta)
\end{aligned}
$$

puisqu'il y a respectivement augmentation et diminution des interactions singulets entre positons et électrons 3d. Le rapport

$$
P(\theta)=\frac{N(\theta) \uparrow \downarrow-N(\theta) \uparrow \uparrow}{N(\theta) \uparrow \downarrow+N(\theta) \uparrow \uparrow}
$$

donne pour chaque valeur de $\theta$, donc de $P_{z}$, une mesure de la densité de spin. En fait, une véritable mesure demanderait, là encore, la connaissance des fonctions d'onde du positon et des électrons.

Le point intéressant est que $P(\theta)>0$ pour $\theta>\theta_{\mathrm{F}}$, c'est-à-dire pour la contribution de caur, ce qui confirme la participation des électrons $3 \mathrm{~d}$ à cette contribution de cœur (alliages de fer, nickel, zinc, cuivre [13]).

Dans certains cas, comme le fer monocristallin, on a obtenu $P(\theta)<0$ pour $0<\theta<\theta_{\mathrm{F}}$, ce qui semble indiquer une polarisation des électrons $4 \mathrm{~s}$ antiparallèlement aux électrons $3 \mathrm{~d}$.

La fusion ne modifie pas la contribution des électrons de cœur, alors que la distribution des électrons de conduction est considérablement modifiée (Fig. 12).

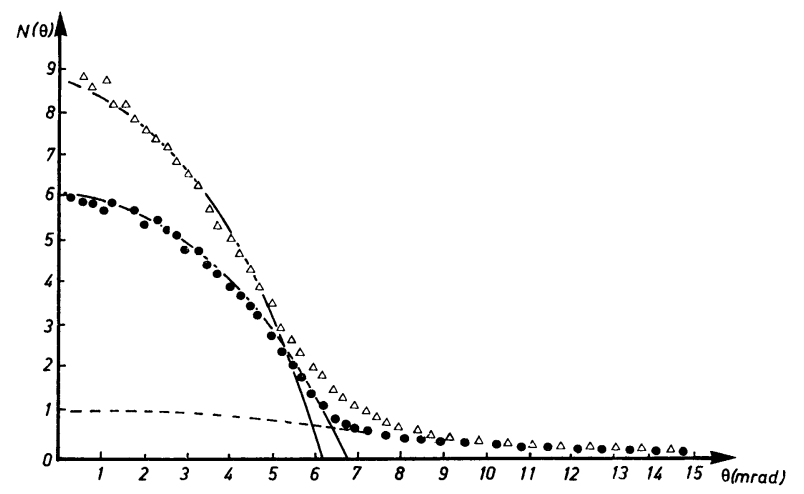

FIG. 12. - Modification de la distribution angulaire des photons d'annihilation par fusion du matériau : $\triangle$ gallium liquide $(320 \mathrm{~K}), \bullet$ gallium solide $(70 \mathrm{~K})$.

3.3.5 Anisotropie dans l'espace des moments. $\mathrm{Si}$ les mesures de corrélations angulaires sont faites sur des monocristaux, l'amplitude de la courbe $N_{(i j k)}\left(P_{z}\right)$ caractérisera un plan cristallin d'indice $(i j k)$ perpendiculaire à la direction d'observation $P_{z}$, et sera proportionnelle à la section correspondante d'une image de la surface de Fermi. Ces images doivent en effet être pondérées par un terme qui dépend $\mathrm{du}$ produit des fonctions d'onde du positon et de l'électron, ce qui introduit une certaine distorsion inévitable avec une technique qui explore l'espace des moments et non pas l'espace des $\mathbf{k}$.

Pour mieux cerner la structure, il convient également d'améliorer la résolution verticale en remplaçant les fentes de grande dimension du banc de corrélation angulaire par des fentes courtes, voire envisager une exploration quasi ponctuelle. Une autre technique consiste à mesurer les corrélations pour $\theta=0$ quand le cristal tourne. Un exemple d'une telle étude est 
illustré par la figure 13 où apparaissent les relations entre courbe de corrélation angulaire et structure de la surface de Fermi dans le cuivre. Il y a en particulier la possibilité de mesurer le diamètre du neck dans la direction $<111>$.

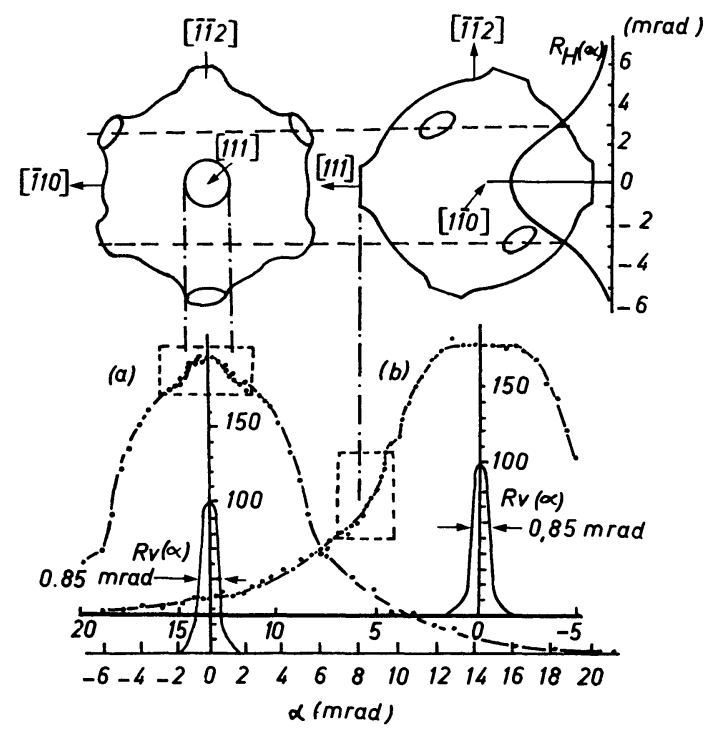

FIG. 13. - Relation entre la forme de la surface de Fermi et la distribution angulaire des photons d'annihilation. Exemple du cuivre.

La technique est particulièrement intéressante pour l'étude des alliages qui ne peuvent être approchés par les moyens précis usuels, tel l'effet Haas-Van Halphen.

La résolution est cependant assez faible et la précision doit être améliorée pour que l'on puisse réellement parler d'exploration quantitative.

4. Application à l'étude des imperfections. - Les imperfections de réseau d'un solide, dans le sens large du terme, peuvent constituer des centres préférentiels d'annihilation pour les positons.

C'est le cas des lacunes, des dislocations, des impuretés de valence plus faible dans les métaux, des surfaces, des cavités, des états électroniques ou magnétiques localisés en général, des zones de désordre, des microprécipités ou inclusions, des centres colorés dans les cristaux ioniques. On dit que le positon s'annihile dans un état piégé. Le mécanisme de piégeage est en général assez simple à comprendre. Dans le cas d'une lacune dans un métal par exemple, il apparaît une région du solide où manque un ion positif, donc où un centre de répulsion du positon a été supprimé : le positon s'annihilera donc préférentiellement sur la lacune; la densité électronique est plus faible qu'ailleurs puisque notamment la contribution de cœur a disparu. Les caractéristiques d'annihilation vont donc se modifier :

- le temps de vie va augmenter,
- la composante parabolique des courbes de corrélation angulaire va croître en valeur relative. On caractérisera cette variation par le paramètre

$$
h=\frac{\text { hauteur du pic }}{\text { aire totale }}(h \text { augmente) }
$$

- la distribution en énergie va s'affiner. Le paramètre de mesure sera

$$
S=\frac{\text { hauteur du pic }}{\text { aire totale }}(S \text { augmente }) .
$$

Les spectres de temps de vie, en particulier, vont alors comporter plusieurs composantes : celles correspondant à l'annihilation dite libre sur les sites normalement occupés, celles correspondant aux annihilations dites piégées sur les sites où se trouvent les défauts. Les problèmes de l'analyse du spectre en ces composantes sont souvent difficiles à résoudre avec précision, car les différents temps de vie sont assez voisins les uns des autres. Nous allons détailler quelque peu le cas de la lacune dans les métaux, à titre d'exemple.

Si $n_{1}(t)$ et $n_{\mathrm{d}}(t)$ sont respectivement la fraction de positons libres et piégés sur le défaut à l'instant $t$, leurs variations dans le temps sont solutions des équations :

$$
\left.\begin{array}{l}
\frac{\mathrm{d} n_{l}(t)}{\mathrm{d} t}=-\frac{n_{l}(t)}{\tau_{l}}-\mu_{\mathrm{d}} c_{\mathrm{d}} n_{l}(t)+v_{\mathrm{d}} n_{\mathrm{d}}(t) \\
\frac{\mathrm{d} n_{\mathrm{d}}(t)}{\mathrm{d} t}=-\frac{n_{\mathrm{d}}(t)}{\tau_{\mathrm{d}}}+\mu_{\mathrm{d}} c_{\mathrm{d}} n_{\mathrm{l}}(t)-v_{\mathrm{d}} n_{\mathrm{d}}(t) .
\end{array}\right\}
$$

La première équation exprime que la réduction de la fraction de positon libre provient de l'annihilation et du piégeage, sous déduction d'une croissance provenant d'un dépiégeage thermiquement activé. La seconde équation exprime que la réduction de la fraction de positon piégé sur les défauts provient de l'annihilation et du dépiégeage thermiquement activé, sous déduction d'une croissance provenant du piégeage statistique ; $\tau_{l}$ et $\tau_{\mathrm{d}}$ sont les temps de vie respectifs pour l'annihilation libre et piégée ; $c_{\mathrm{d}}$ est la concentration de défauts piégeants; $\mu_{\mathrm{d}}$ est la vitesse spécifique de piégeage par les défauts ; $v_{d}$ est la vitesse de dépiégeage. Le terme de dépiégeage peut être estimé par une relation du type

$$
v_{\mathrm{d}} \simeq \frac{1}{a} \sqrt{\frac{k T}{2 \pi m}} \exp \left(-\frac{\varepsilon_{\mathrm{dp}}}{k T}\right)
$$

où $\varepsilon_{\mathrm{dp}}$ est l'énergie de liaison défaut-positon, $a$ le rayon d'une sphère caractérisant le volume où le positon est piégé, $m$ la masse d'un électron. Le dépiégeage sera négligeable si l'annihilation est plus rapide, donc si :

$$
v_{\mathrm{d}}<\frac{1}{\tau_{\mathrm{d}}}
$$


soit

$$
\varepsilon_{\mathrm{dp}}>\frac{k T}{2} \log \left(\frac{k T \tau_{\mathrm{d}}^{2}}{2 \pi m a^{2}}\right) .
$$

Si l'on prend $a \simeq 2 \times 10^{-8} \mathrm{~cm}$ et $\tau_{\mathrm{d}} \simeq 2 \times 10^{-10} \mathrm{~s}$, on trouve qu'il est nécessaire que $\varepsilon_{\mathrm{dp}}>0,25 \mathrm{eV}$ à $300 \mathrm{~K}$ et $\varepsilon_{\mathrm{dp}}>0,90 \mathrm{eV}$ à $1000 \mathrm{~K}$.

Des estimations faites par Hodges [14] donnent des valeurs de $\tau_{\mathrm{dp}}$ comprises entre 0,13 et $1 \mathrm{eV}$ pour les alcalins, entre $0,8 \mathrm{eV}$ et $2,4 \mathrm{eV}$ pour les métaux hexagonaux, entre $1,8 \mathrm{eV}$ et $3,7 \mathrm{eV}$ pour l'aluminium. Ces chiffres peuvent expliquer pourquoi on n'a pas pu mettre en évidence le piégeage par les lacunes dans les métaux alcalins. Pour les autres métaux, le dépiégeage semble faible, mais peut-être pas entièrement négligeable à haute température. C'est un point qui mériterait d'être étudié plus en détail.

$\mathrm{La}$ résolution du système (ix) se fait habituellement en posant $v_{\mathrm{d}}=0$; on obtient alors l'expression du taux d'annihilation en fonction du temps [15]

$$
\begin{aligned}
P(t) & =\frac{\mathrm{d}}{\mathrm{d} t}\left[n_{l}(t)+n_{\mathrm{d}}(t)\right] \\
& =\frac{\tau_{l} \mu_{\mathrm{d}} c_{\mathrm{d}}}{\tau_{\mathrm{d}}-\tau_{l}+\tau_{\mathrm{d}} \tau_{l} \mu_{\mathrm{d}} c_{\mathrm{d}}} \exp \left(-\frac{t}{\tau_{\mathrm{d}}}\right)+ \\
& +\frac{\left(\tau_{\mathrm{d}}-\tau_{l}\right)\left[1 / \tau_{l}+\mu_{\mathrm{d}} c_{\mathrm{d}}\right]}{\tau_{\mathrm{d}}-\tau_{l}+\tau_{\mathrm{d}} \tau_{l} \mu_{\mathrm{d}} c_{\mathrm{d}}} \exp \left(-t\left[\frac{1}{\tau_{l}}+\mu_{\mathrm{d}} c_{\mathrm{d}}\right]\right) .
\end{aligned}
$$

On obtient donc un spectre à deux composantes, dont l'une est caractérisée par un temps de vie dépendant de la concentration $c_{\mathrm{d}}$ en défaut.

Dans le cas des lacunes, on fait varier $c_{\mathrm{d}}$ en augmentant la température. L'analyse des spectres permet donc de déterminer $\mu_{\mathrm{d}} c_{\mathrm{d}}(T)$ et, ensuite, les paramètres thermodynamiques caractéristiques de la lacune. Le formalisme peut en principe s'appliquer à tout autre centre piégeant, et à plusieurs centres piégeants existant simultanément. Depuis quelques temps, de nombreuses études de ce type se sont développées et constituent certainement la voie d'avenir pour l'utilisation de l'annihilation de positon en physique du solide.

Certains problèmes restent encore à préciser cependant. En particulier, les résultats numériques dépendent encore beaucoup de la méthode d'exploitation des spectres ; ceci est dû à la précision insuffisante des mesures. La variation linéaire des paramètres $h$ et $S$ en fonction de la contribution relative des différents types d'annihilation, du type

$$
\bar{h}=\frac{I_{l} h_{l}+I_{\mathrm{d}} h_{\mathrm{d}}}{I_{l}+I_{\mathrm{d}}}
$$

n'est pas non plus une hypothèse très solide, car les distributions angulaires et en énergie sont assez sensibles aux détails des états des électrons et du positon. Enfin, les variations éventuelles des vitesses de piégeage $\mu_{\mathrm{d}}$, des constantes $\tau_{\mathrm{l}}, \tau_{\mathrm{d}}, h_{1}, h_{\mathrm{d}}, S_{1}$ et $S_{\mathrm{d}}$, notamment avec la température dans le cas des lacunes, sont pratiquement impossibles à définir avec sûreté ; la mise au point de modèle précis reste à faire.

La méthode possède par ailleurs certaines originalités qui en font un outil précieux. Le piégeage total des positons dès que la concentration en lacunes atteint $10^{-5}$ permet de rester à température relativement basse et d'être assuré d'observer un phénomène dû aux monolacunes. L'apparition de défauts plus étendus (bilacunes par exemple) doit se traduire d'ailleurs par un deuxième stade de l'augmentation du paramètre moyen d'annihilation [16], d'où une possibilité de distinguer entre types de défauts différents et de préciser par exemple un mécanisme de diffusion, ou l'évolution de défauts au cours d'une opération de trempe, ou un phénomène de rassemblement en petits amas.

Le champ des applications de l'annihilation des positons, en particulier pour l'étude des états de surface, ne devrait pas manquer de s'accroître dans les prochaines années.

\section{Bibliographie}

[1] West, R. N., Adv. Phys. 22 (1973) no 3.

[2] Seeger, A., J. Phys. F. 3 (1973) 248.

[3] Doyama, M., Hasiguti, R. R., Cryst. Lattice Defects 4 (1973) 139.

[4] Dekhtyar, I. Ya., Physics Report (Phys. Lett. C) 9C (1974) 245.

[5] GoldanskiI, V. I., Revue d'Energie Atomique (Ed. par I. A. E. A.) $6 \mathrm{n}^{\circ} 1$ (1968).

[6] Stewart, A. T., Roellig, L. O., Positron Annihilation (Academic Press-New York et London) 1967.

[7] Kahana, S., Phys. Rev. 129 (1963) 1622.
[8] Carbotte, J.-P., Phys. Rev. 155 (1967) 197.

[9] SJölander, A. et Stott, M. J., Phys. Rev. B 5 (1972) 2109.

[10] Bhattacharyya, F. et Singwi, K. S., Phys. Rev. Lett. 29 (1972) 22.

[11] Stott, M. J. et Kubica P., Phys. Rev. B 11 (1975) 1.

[12] Brandt, W. et Spirn, I., Phys. Rev. 142 (1966) 231.

[13] Mijnarends, P. E., Physica 63 (1973) 248.

[14] Hodges, C. H., Phys. Rev. Lett. 25 (1970) 284.

[15] Hall, T. M., Goland, A. N. et Snead Jr., C. L., Phys. Rev. B 10 (1974) 3062.

[16] Sueoka, O., J. Phys. Soc. Japan 36 (1974) 464. 